\title{
Therapeutic Efficacy Evaluation of 111 In-Labeled PEGylated Liposomal Vinorelbine in Murine Colon Carcinoma with Multimodalities of Molecular Imaging
}

\author{
Tong-Hsien Chow ${ }^{1}$, Yi-Yu Lin ${ }^{1}$, Jeng-Jong Hwang ${ }^{1}$, Hsin-Ell Wang ${ }^{1}$, Yun-Long Tseng ${ }^{2}$, Victor Fei Pang ${ }^{3}$, \\ Ren-Shyan Liu ${ }^{4}$, Wuu-Jyh Lin ${ }^{5}$, Chung-Shi Yang ${ }^{6}$, and Gann Ting 6
}

${ }^{1}$ Department of Biomedical Imaging and Radiological Sciences, National Yang-Ming University, Taipei, Taiwan; ${ }^{2}$ Taiwan Liposome Company, Taipei, Taiwan; ${ }^{3}$ Department of Veterinary Medicine, National Taiwan University, Taipei, Taiwan; ${ }^{4}$ Department of Nuclear Medicine, Taipei Veterans General Hospital, Taipei, Taiwan; ${ }^{5}$ Institute of Nuclear Energy Research, Taoyuan, Taiwan; and ${ }^{6}$ National Health Research Institute, Taipei, Taiwan

\begin{abstract}
In our previous studies using combined radioisotopes with chemotherapeutic liposomal drugs (i.e., ${ }^{111}$ In-labeled polyethylene glycol (PEG)ylated liposomal vinorelbine) we have reported possible therapeutic efficiency in tumor growth suppression. Nevertheless, the challenge remains as to whether this chemotherapy has a therapeutic effect as good as that of combination therapy. The goal of this study was to investigate the real therapeutic effectiveness of 6 mol\% PEG ${ }^{111}$ In-vinorelbine liposomes via the elevation of the radiation dosage and reduction in the concentration of chemotherapeutic agents. Methods: Murine colon carcinoma cells transfected with dual-reporter genes (CT-26/tk-luc) were xenografted into BALB/c mice. The biodistribution was estimated to determine the drug profile and targeting efficiency of ${ }^{111} \mathrm{In}$-vinorelbine liposomes. Bioluminescence imaging and ${ }^{18} \mathrm{~F}-$
\end{abstract} FDG small-animal PET were applied to monitor the therapeutic response after drug administration. The survival in vivo was estimated and linked with the toxicologic and histopathologic analyses to determine the preclinical safety and feasibility of the nanomedicine. Results: Effective long-term circulation of radioactivity in the plasma was achieved by 6 mol\% PEG ${ }^{111}$ In-vinorelbine liposomes, and this dose showed significantly lower uptake in the reticuloendothelial system than that of $0.9 \mathrm{~mol} \%$ PEG ${ }^{111}$ In-vinorelbine liposomes. Selective tumor uptake was represented by cumulative deposition, and the maximum accumulation was at $48 \mathrm{~h}$ after injection. The combination therapy exhibited an additive effect for tumor growth suppression as tracked by caliper measurement, bioluminescence imaging, and small-animal PET. Furthermore, an improved survival rate and reduced tissue toxicity were closely correlated with the toxicologic and histopathologic results. Conclusion: The results demonstrated that the use of 6 mol\% PEG ${ }^{111}$ In-vinorelbine liposomes for passively targeted tumor therapy displayed an additive effect with combined therapy, not only by prolonging the circulation rate because of a reduction in the phagocytic effect

Received Feb. 22, 2009; revision accepted Aug. 28, 2009.

For correspondence or reprints contact: Jeng-Jong Hwang, Department of Biomedical Imaging and Radiological Sciences, National Yang-Ming University, 155 Li-Nong St., Section 2, Pei-tou, Taipei 112, Taiwan.

E-mail: jjhwang@ym.edu.tw

COPYRIGHT ( 2009 by the Society of Nuclear Medicine, Inc. of the reticuloendothelial system but also by enhancing tumor uptake. Thus, this preclinical study suggests that $6 \mathrm{~mol} \%$ PEG

${ }^{111}$ In-vinorelbine liposomes have the potential to increase the therapeutic index and reduce the toxicity of the passively nanotargeted chemoradiotherapies.

Key Words: ${ }^{111}$ In-vinorelbine liposomes; CT-26/tk-luc; bioluminescence imaging (BLI); ${ }^{18} \mathrm{~F}-\mathrm{FDG}$ small-animal PET; reticuloendothelial system (RES)

J Nucl Med 2009; 50:2073-2081

DOI: 10.2967/jnumed.109.063503

\section{L} iposomes are polymeric nanoparticles that consist of phospholipid bilayers and generate an aqueous cavity in the inner phase, which can be a stable shelter for pharmacologic agents that include chemotherapeutic drugs used in cancer therapy, antisense oligonucleotides used in gene therapy, peptides used in infectious disease treatments, antigens that stimulate an immune response, and radiopharmaceuticals used for targeting diagnosis and therapy (1-3). These encapsulated agents exhibit stable pharmacokinetics, a better distribution in human bodies, and reduced tissue toxicity and are mainly delivered to the regions of interest (ROIs) - all or which are characteristics that improve therapeutic effectiveness $(4,5)$.

One defect of traditional liposomes is that they are easily taken up by cells of the mononuclear phagocyte system, primarily those located in the reticuloendothelial system (RES) (6). Polyethylene glycol (PEG) modification of the liposomes (PEGylated liposomes) was initially developed to resist opsonization, evade rapid clearance by the RES, and allow the liposomes to remain in the circulation for a prolonged period after systemic administration $(6,7)$. The PEGylated liposomal formulation of doxorubicin has been applied as a treatment for AIDS-related Kaposi sarcoma 
$(8,9)$, ovarian carcinoma $(10,11)$, breast carcinoma $(12,13)$, mullerian carcinoma (14), and prostate cancer $(15,16)$. Furthermore, this approach has gained regulatory approval for use as a clinical therapy $(8-13)$.

Recently, we have reported that the intravenous administration of $6 \mathrm{~mol} \%$ PEGylated liposomes was able to not only prolong the circulating rate of the liposomes but also reduce the amount of radioactivity accumulating in the RES (17). Furthermore, the increase in the tumor-targeting efficiency of this treatment, compared with the $0.9 \mathrm{~mol} \%$ treatment, suggests that the concentration of PEGylation influences the spatial and temporal distribution of liposomal drugs. In addition, we have further demonstrated that 6 mol\% PEGylated liposomes encapsulated with an ${ }^{111}$ In radionuclide and the anticancer drug vinorelbine have a potent therapeutic effect in tumor growth inhibition (3). These results provide important information with regard to the formulation of $6 \mathrm{~mol} \%$ PEG ${ }^{111} \mathrm{In}$-vinorelbine liposomes that may be a suitable drug delivery system for cancer diagnosis using $\gamma$-rays and for cancer therapy using vinorelbine and Auger electrons.

In our previous study, vinorelbine liposomes displayed a therapeutic effect similar to that of ${ }^{111}$ In-vinorelbine liposomes (3). Therefore, to clarify whether the combined therapy of ${ }^{111}$ In-vinorelbine liposomes gave either an additive or a synergistic effect, compared with ${ }^{111} \mathrm{In}$ liposomes or vinorelbine liposomes alone, the radiation dosage of ${ }^{111}$ In was increased to $37 \mathrm{MBq}$ and the concentration of vinorelbine was decreased to $3 \mathrm{mg} / \mathrm{kg}$ in this therapeutic trial.

\section{MATERIALS AND METHODS}

\section{Tumor Cell Preparation}

CT-26/tk-luc tumor cells were maintained in RPMI 1640 medium (Gibco-BRL), with $10 \%$ heat-inactivated fetal bovine serum (Hyclone) supplemented with L-glutamine, sodium bicarbonate, 100 units of penicillin per milliliter, and $100 \mu \mathrm{g}$ of streptomycin per milliliter at $37^{\circ} \mathrm{C}$ with $5 \% \mathrm{CO}_{2}$ in the presence of $500 \mu \mathrm{g}$ of G418 per milliliter (Merck), to maintain stable expression of the reporter genes.

\section{Tumor-Xenografted Animal Model}

Tumor cells $\left(2 \times 10^{6}\right.$ cells $\left./ 200 \mu \mathrm{L}\right)$ were transplanted subcutaneously into the dorsal region of the right thigh of BALB/c mice (purchased from the National Laboratory Animal Center). Subcutaneous transplantation was followed by daily digital caliper measurement of tumor growth once the bulge caused by the tumor cells at the injection site was visible $14 \mathrm{~d}$ after inoculation (tumor size, $\sim 50 \pm 5 \mathrm{~mm}^{3}$ ). The tumor volume was calculated using the formula $0.523 \times($ length $\times$ width $\times$ thickness $)$ and assessed twice per week for $1 \mathrm{mo}$.

\section{Preparation of PEGylated Liposomes}

The PEGylated liposomes were prepared as previously described by Tseng et al. (18). Briefly, PEG-distearoylphosphatidylethanolamine (DSPE) (0.9 and $6 \mathrm{~mol} \%$ ) was prepared using the following ratios: distearoylphosphatidylcholine:cholesterol:DSPE covalently linked PEG of 2:1:0.027 for the $0.9 \mathrm{~mol} \%$ and of
2:1:0.18 for the $6 \mathrm{~mol} \%$ PEG-DSPE. Small unilamellar vesicles (standardized uptake value [SUV], $\sim 100-\mathrm{nm}$ diameter) were produced by a combination of the standard thin-film hydration method, the freeze-and-thaw method, and repeated extrusion. The extraliposomal salt was removed by a Sephadex G-50 column (Bio-Rad) and elution with histidine-sucrose buffer ( $\mathrm{pH}$ 6.0).

\section{Anticancer Drug Encapsulation}

After the extraliposomal salt was removed by a Sephadex G-50 column, the anticancer agent vinorelbine was added immediately into the solution at a concentration of $3.5 \mathrm{mg}$ of vinorelbine per $10 \mu \mathrm{mol}$ of phospholipid and incubated at $60^{\circ} \mathrm{C}$ for $30 \mathrm{~min}$ with agitation (100 rpm). After loading, the liposomal vinorelbine was sterilized by $0.2-\mu \mathrm{m}$ filtration and stored at $4{ }^{\circ} \mathrm{C}-6^{\circ} \mathrm{C}$ until use. The PEGylated liposomes (NanoVNB; Taiwan Liposome Company) were characterized as follows: $\mathrm{pH}, 6.1$; osmolarity, 361 $\mathrm{mmol} / \mathrm{kg}$; mean particle size, $95.2 \mathrm{~nm}$; phospholipids, $6.19 \mu \mathrm{mol} /$ $\mathrm{mL}$; and vinorelbine, $2.08 \mathrm{mg} / \mathrm{mL}$.

\section{Radiolabeling of ${ }^{111}$ In-Oxine}

Oxine was labeled with ${ }^{111} \mathrm{In}$ as previously described by Chow et al. (3). Briefly, $15 \mu \mathrm{L}$ of $68 \mathrm{mM}$ 8-hydroxyquinoline (oxine; Sigma-Aldrich Co.) in ethanol were added to $10 \mu \mathrm{L}$ of ${ }^{111} \mathrm{In}$ (indium chloride in $0.05 \mathrm{M} \mathrm{HCl}$; 3.7-74 MBq) (Perkin Elmer) in $400 \mu \mathrm{L}$ of $0.1 \mathrm{M}$ sodium acetate buffer $(\mathrm{pH}$ 5.5) and then incubated at $50^{\circ} \mathrm{C}$ for $20 \mathrm{~min}$. The lipophilic components were extracted with chloroform and then evaporated. The labeling efficiency of ${ }^{111} \mathrm{In}$-oxine was determined by an instant thin-layer chromatography method. The radiochemical yield was generally greater than $90 \%{ }^{111}$ In-oxine.

\section{Preparation of PEGylated ${ }^{111}$ In-Vinorellbine Liposomes}

The extracted ${ }^{111}$ In-oxine residue was dissolved in $20 \mu \mathrm{L}$ of ethanol and added to $80 \mu \mathrm{L}$ of distilled water. The mixture was incubated with $1.5 \mathrm{~mL}$ of liposomes for $30 \mathrm{~min}$ at $37^{\circ} \mathrm{C}$. Ethylenediaminetetraacetic acid ( $2 \mathrm{mg})$ was then added to chelate any residual free ${ }^{111} \mathrm{In}$ and to promote prompt excretion after intravenous injection. The labeling of ${ }^{111}$ In within the liposomes (0.9 and $6 \mathrm{~mol} \%$ PEG) was assayed by loading a $100-\mu \mathrm{L}$ sample onto a column $(40 \times 8 \mathrm{~mm})($ Bio-Rad) containing Sephadex G-50 fine gel (Amersham Biosciences) and elution was with normal saline. The labeling efficiency was determined by dividing the radioactivity from vinorelbine liposome fractions by the total amount loaded. The radioactivity of each fraction was measured using either a dose calibrator (CRC-15R, Capintec; Bioscan) or a $\gamma$-scintillation counter (Cobra II Auto- $\gamma$-counter; Packard). The entrapment of ${ }^{111} \mathrm{In}$ was more than $90 \%$ (3).

\section{Biodistribution of ${ }^{111}$ In-Vinorelbine Liposomes in CT-26/tk-luc Tumor-Bearing Mice}

The tumor-bearing mice (tumor size, $\sim 70 \pm 10 \mathrm{~mm}^{3}$ ) were randomly divided into 3 groups; each mouse received an intravenous administration of $3.7 \mathrm{MBq}$ of $0.9 \mathrm{~mol} \%$ PEG ${ }^{111} \mathrm{In}-$ vinorelbine liposomes, 6 mol\% PEG ${ }^{111}$ In-vinorelbine liposomes, or unencapsulated ${ }^{111} \mathrm{In}-1,4,7,10$-tetraazacyclododecane- $N, N^{\prime}, N^{\prime \prime}$, $N^{\prime \prime \prime}$-tetraacetic acid (DOTA). For the biodistribution studies $(n=4$ per time point), mice were sacrificed at $1,4,24,48$, and $72 \mathrm{~h}$ after injection. Anatomization was performed, and tissues and organs of interest-including the blood, heart, lungs, liver, stomach, spleen, pancreas, large and small intestines, bladder, urine, kidneys, muscle, bone marrow, and tumor-were excised. The net tissue weights were obtained, and the level of radioactivity in each tissue was measured 
using a $\gamma$-scintillation counter at $72 \mathrm{~h}$ after injection. The results were expressed as counts per minute with decay correction standards and were normalized as percentage of the injected dose per gram of tissue $(\% \mathrm{ID} / \mathrm{g})(19)$.

\section{Bioluminescence Imaging (BLI)}

BLI was performed using an IVIS50 animal imaging system (Xenogen Corp.). The photons emitted from the target site penetrated through the mammalian tissue and could be externally detected and quantified using a sensitive light-imaging system (3). On day 17 after inoculation (tumor size, $70 \pm 5 \mathrm{~mm}^{3}$ ), the mice were anesthetized with $1 \%-3 \%$ isoflurane using a vaporizer system and intraperitoneally injected with $150 \mathrm{mg}$ of D-luciferin per kilogram at $15 \mathrm{~min}$ before imaging. The image acquisition time was $1 \mathrm{~min}$. The displayed images of the tumor sites were drawn around and quantified in photons per second using Living Image software (Xenogen Corp.).

\section{${ }^{18}$ F-FDG Small-Animal PET}

${ }^{18}$ F-FDG was provided by the National PET and Cyclotron Center at Taipei Veterans General Hospital. On day 17 after inoculation (tumor size, $\sim 70 \pm 5 \mathrm{~mm}^{3}$ ), the conscious mice were intravenously injected with $6.29 \pm 0.37 \mathrm{MBq}$ of ${ }^{18} \mathrm{~F}-\mathrm{FDG}$, and 30 min was allowed for systemic uptake. Fully 3-dimensional listmode emission data were obtained using a microPET R4 scanner (Concorde Microsystems) and collected from 30 to 60 min after radiotracer injection. The 3-dimensional whole-body images of mice were obtained using a system that produced 63 image slices over $10.5 \mathrm{~cm}$ of $x$ and $y$ and $7.8 \mathrm{~cm}$ of $z$ axial fields of view. The spatial resolution was $4.6 \mathrm{~mm}$ in the central axis using the 2-dimensional mode. No attenuation correction was performed in this research. Finally, a cylinder calibration method was used to convert the units of the small-animal PET images from counts per second per voxel to nanocuries per cubic centimeter. The radioactivity $\left(37 \mathrm{~Bq} / \mathrm{cm}^{3}\right), \% \mathrm{ID} / \mathrm{g}$, and SUV of the ROIs of the tumor were drawn and calculated at various times from all images.

\section{Body Weight and Survival Assessment}

The body weight losses and surviving fractions were estimated after a single dose of drug was administered. The body weight change of each mouse was measured twice per week for 1 mo. Survival was monitored daily and continued until all mice were dead after injection. The results then underwent Kaplan-Meier survival analysis.

\section{Biochemistry and Hematology Analyses}

At the completion of the experiments (at the fifth week after drug administration), the mice were sacrificed. Blood samples were collected and tested for levels of white blood cells, red blood cells, hemoglobin, hematocrit, and platelets. Serum was collected and analyzed for levels of alkaline phosphatase (ALP), albumin, alanine aminotransferase (ALT), aspartate aminotransferase, creatinine, and blood urea nitrogen (BUN). These measurements were performed on a Beckman CX-5 PRO.

\section{Tissue Preparation for Histopathology}

At the end of the experiments, samples of the tumor mass and the major organs including the lungs, liver, spleen, and kidneys were fixed in cold $4 \%$ paraformaldehyde, embedded in paraffin, sectioned at $5 \mu \mathrm{m}$, and stained with hematoxylin and eosin.

\section{Statistical Analysis}

The Student $t$ test was used to analyze for significant differences between the control and drug-treated mice. $P$ values of less than 0.05 and 0.01 were defined as the significance levels.

\section{RESULTS}

\section{Biodistribution of ${ }^{111}$ In-Vinorelbine Liposomes}

in CT-26/tk-luc Tumor-Bearing Mice

The accumulation of radioactivity in the tissues of the CT-26/tk-luc tumor-bearing BALB/c mice $(n=4)$ was measured at the times described after a $3.7 \mathrm{MBq} / 100 \mu \mathrm{L}$ intravenous administration of 0.9 or $6 \mathrm{~mol} \%$ PEG ${ }^{111} \mathrm{In}$ vinorelbine liposomes or unencapsulated ${ }^{111}$ In-DOTA. In Figure 1, the concentration of radioactivity in the blood decreased rapidly from $34.74 \pm 9.15 \% \mathrm{ID} / \mathrm{g}$ to $0.25 \pm 0.07$ $\%$ ID/g after treatment with 0.9 mol\% PEG ${ }^{111}$ In-vinorelbine liposomes, compared with 6 mol\% (from $27.91 \pm 0.98$ $\% \mathrm{ID} / \mathrm{g}$ to $7.67 \pm 0.13 \% \mathrm{ID} / \mathrm{g})$. However, $6 \mathrm{~mol} \% \mathrm{PEG}$ ${ }^{111}$ In-vinorelbine liposomes significantly decreased the radioactivity accumulation in the liver, spleen, and bone marrow and gave a better retention in the tumor than did $0.9 \mathrm{~mol} \%$. There was no significant difference in renal uptake between the groups. Selective tumor uptake was measured either by the cumulative deposition and the maximum accumulation $(14.92 \pm 3.96 \% \mathrm{ID} / \mathrm{g})$ or by the tumor-tomuscle ratio (16.76) at $48 \mathrm{~h}$ after injection (Table 1). In addition, a rapid clearance of radioactivity was found with the ${ }^{111}$ In-DOTA treatment, with most radioactivity being excreted through the urinary system (Table 2). These results suggest that circulation time and tumor-targeting capability were enhanced, and the RES phagocytic function was reduced when 6 mol\% PEGylated liposomes were used.

\section{BLI for Monitoring Therapeutic Response}

Therapeutic responses were monitored by BLI (Fig. 2A) and digital caliper measurement (Fig. 3) before and twice a week after drug treatment. The greatest suppression of tumor growth was found with the combination therapy using ${ }^{111}$ In-vinorelbine liposomes. A moderate inhibition was found with the chemotherapy using vinorelbine liposomes, and this was followed by radiotherapy with ${ }^{111}$ In-liposomes. In this study, both the ${ }^{111}$ In-DOTA (without liposomal encapsulation) and NanoX liposome groups (empty liposomes without ${ }^{111} \mathrm{In}$ and vinorelbine) were used as controls for the comparison. The photon counts from the BLI were collected and measured from the ROIs of the tumor sites. The mean photon flux of all the treatments correlated with tumor size (Fig. 2B). The results showed that mean photon flux rapidly increased with tumor volume up to about 800$1,000 \mathrm{~mm}^{3}$, then gradually decreased. The latter effect may be because the larger tumors tended to have significant necrosis, which does not emit bioluminescence and affects the results relative to smaller tumors. In these circumstances, fewer photon counts would be present for the same ROI. This possibility was later confirmed by examination of the pathology of the tumors. The mean photon fluxes, as 


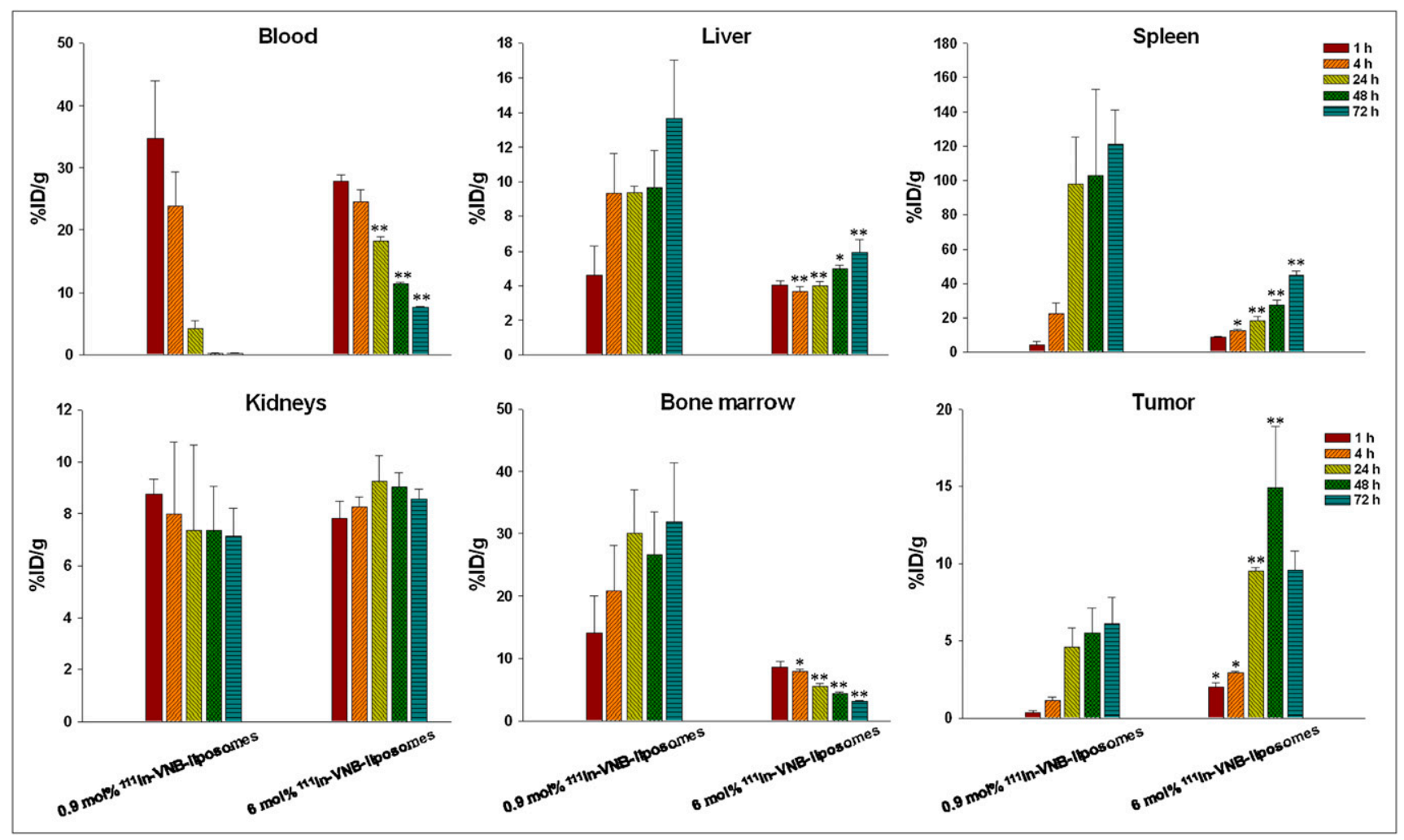

FIGURE 1. Biodistribution of ${ }^{111}$ In-labeled 0.9 mol\% and 6 mol\% PEG ${ }^{111}$ In-vinorelbine liposomes in CT-26/tk-luc tumorbearing BALB/c mice. Tissue uptake is expressed as $\% \mathrm{ID} / \mathrm{g}$ (mean $\pm \mathrm{SE}, n=4$ ). Experiments were repeated 4 times. ${ }^{*} P<0.05$ and ${ }^{\star \star} P<0.01$ are significantly different between both groups by Student $t$ test.

a function of time after initiation of the various treatments, are shown in Figure 2C, which uses the BLI data from before day 40. These results confirm that among all the treatments, the greatest tumor control was given by combination therapy, followed by chemotherapy and then radiotherapy.

\section{${ }^{18}$ F-FDG Small-Animal PET for Monitoring \\ Therapeutic Response}

${ }^{18}$ F-FDG small-animal PET was performed before and once per week after the various treatments. The coronal views of the small-animal PET images in Supplemental

\begin{tabular}{|c|c|c|c|c|c|}
\hline \multirow[b]{2}{*}{ Organ } & \multicolumn{5}{|c|}{ Time after intravenous administration (h) } \\
\hline & 1 & 4 & 24 & 48 & 72 \\
\hline Blood & $27.91 \pm 0.98$ & $24.48 \pm 2.04$ & $18.23 \pm 0.67$ & $11.41 \pm 0.23$ & $7.67 \pm 0.13$ \\
\hline Heart & $5.91 \pm 0.73$ & $4.22 \pm 0.13$ & $3.29 \pm 0.16$ & $3.12 \pm 0.33$ & $3.46 \pm 0.40$ \\
\hline Lungs & $12.50 \pm 1.45$ & $13.79 \pm 1.30$ & $11.92 \pm 1.49$ & $10.25 \pm 1.27$ & $6.31 \pm 0.79$ \\
\hline Liver & $4.02 \pm 0.25$ & $3.67 \pm 0.28$ & $3.99 \pm 0.25$ & $5.00 \pm 0.20$ & $5.92 \pm 0.71$ \\
\hline Stomach & $2.27 \pm 0.31$ & $2.17 \pm 0.27$ & $3.99 \pm 0.96$ & $3.80 \pm 0.36$ & $2.15 \pm 0.25$ \\
\hline Spleen & $8.73 \pm 0.40$ & $12.45 \pm 0.72$ & $18.34 \pm 2.21$ & $27.52 \pm 2.94$ & $44.92 \pm 2.60$ \\
\hline Pancreas & $2.12 \pm 0.17$ & $2.36 \pm 0.31$ & $1.89 \pm 0.13$ & $1.76 \pm 0.08$ & $1.38 \pm 0.11$ \\
\hline Large intestine & $2.08 \pm 0.31$ & $2.63 \pm 0.71$ & $5.57 \pm 1.38$ & $7.20 \pm 1.51$ & $6.93 \pm 1.87$ \\
\hline Small intestine & $5.43 \pm 0.83$ & $7.68 \pm 1.10$ & $9.26 \pm 0.99$ & $12.84 \pm 0.30$ & $8.60 \pm 1.68$ \\
\hline Bladder & $2.18 \pm 0.12$ & $2.43 \pm 0.77$ & $1.29 \pm 0.15$ & $1.09 \pm 0.09$ & $1.50 \pm 0.16$ \\
\hline Urine & $1.74 \pm 0.15$ & $0.86 \pm 0.10$ & $0.74 \pm 0.04$ & $0.69 \pm 0.09$ & $0.80 \pm 0.08$ \\
\hline Kidneys & $7.81 \pm 0.67$ & $8.27 \pm 0.37$ & $9.26 \pm 0.99$ & $9.03 \pm 0.55$ & $8.56 \pm 0.38$ \\
\hline Muscle & $1.15 \pm 0.14$ & $0.79 \pm 0.07$ & $0.87 \pm 0.04$ & $0.89 \pm 0.07$ & $1.30 \pm 0.19$ \\
\hline Bone marrow & $8.55 \pm 0.95$ & $7.94 \pm 0.35$ & $5.43 \pm 0.47$ & $4.38 \pm 0.19$ & $3.05 \pm 0.19$ \\
\hline Tumor & $1.97 \pm 0.31$ & $2.88 \pm 0.14$ & $9.55 \pm 0.20$ & $14.92 \pm 3.96$ & $9.60 \pm 1.21$ \\
\hline Tumor/muscle & 1.71 & 3.65 & 10.98 & 16.76 & 7.38 \\
\hline
\end{tabular}


TABLE 2. Biodistribution of CT-26/tk-luc Tumor-Bearing BALB/c Mice After Intravenous Administration of ${ }^{111}$ In-DOTA

\begin{tabular}{|c|c|c|c|c|c|}
\hline \multirow[b]{2}{*}{ Organ } & \multicolumn{5}{|c|}{ Time after intravenous administration (h) } \\
\hline & 1 & 4 & 24 & 48 & 72 \\
\hline Blood & $0.29 \pm 0.05$ & $0.03 \pm 0.01$ & $0.004 \pm 0.0002$ & $0.004 \pm 0.0004$ & $0.003 \pm 0.0001$ \\
\hline Heart & $0.08 \pm 0.01$ & $0.04 \pm 0.01$ & $0.02 \pm 0.006$ & $0.02 \pm 0.006$ & $0.02 \pm 0.005$ \\
\hline Lungs & $0.36 \pm 0.05$ & $0.15 \pm 0.05$ & $0.09 \pm 0.03$ & $0.12 \pm 0.007$ & $0.04 \pm 0.004$ \\
\hline Liver & $0.37 \pm 0.03$ & $0.30 \pm 0.01$ & $0.27 \pm 0.04$ & $0.21 \pm 0.01$ & $0.25 \pm 0.01$ \\
\hline Stomach & $0.13 \pm 0.02$ & $0.06 \pm 0.01$ & $0.02 \pm 0.002$ & $0.02 \pm 0.001$ & $0.01 \pm 0.001$ \\
\hline Spleen & $0.26 \pm 0.02$ & $0.18 \pm 0.03$ & $0.22 \pm 0.01$ & $0.18 \pm 0.002$ & $0.13 \pm 0.01$ \\
\hline Pancreas & $0.09 \pm 0.02$ & $0.03 \pm 0.007$ & $0.01 \pm 0.001$ & $0.01 \pm 0.001$ & $0.01 \pm 0.001$ \\
\hline Large intestine & $0.21 \pm 0.10$ & $0.07 \pm 0.009$ & $0.07 \pm 0.02$ & $0.03 \pm 0.002$ & $0.02 \pm 0.004$ \\
\hline Small intestine & $0.21 \pm 0.06$ & $0.15 \pm 0.03$ & $0.04 \pm 0.01$ & $0.03 \pm 0.004$ & $0.02 \pm 0.003$ \\
\hline Bladder & $137.03 \pm 28.12$ & $4.14 \pm 1.95$ & $0.21 \pm 0.06$ & $0.29 \pm 0.06$ & $0.30 \pm 0.09$ \\
\hline Urine & $467.27 \pm 38.80$ & $131.97 \pm 81.36$ & $0.63 \pm 0.28$ & $0.09 \pm 0.02$ & $0.11 \pm 0.03$ \\
\hline Kidneys & $2.58 \pm 0.50$ & $1.21 \pm 0.18$ & $0.58 \pm 0.03$ & $0.28 \pm 0.05$ & $0.21 \pm 0.02$ \\
\hline Muscle & $0.09 \pm 0.03$ & $0.11 \pm 0.06$ & $0.01 \pm 0.001$ & $0.01 \pm 0.001$ & $0.01 \pm 0.001$ \\
\hline Bone marrow & $0.40 \pm 0.14$ & $0.58 \pm 0.20$ & $0.18 \pm 0.10$ & $0.19 \pm 0.08$ & $0.19 \pm 0.04$ \\
\hline Tumor & $0.25 \pm 0.02$ & $0.17 \pm 0.03$ & $0.06 \pm 0.01$ & $0.02 \pm 0.002$ & $0.02 \pm 0.001$ \\
\hline Tumor/muscle & 2.78 & 1.55 & 6 & 2 & 2 \\
\hline
\end{tabular}

Figure 1A (supplemental materials are available online only at http://jnm.snmjournals.org) show a clear tumor uptake despite the large amounts of radioactivity that had accumulated in the circulatory and urinary systems. The calibration of the ${ }^{18}$ F-FDG metabolism (Supplemental Fig. 1B) showed a linear correlation with tumor size $\left(R^{2}=\right.$ $0.881)$. The quantified results of tumor metabolic activity for all treatments (Supplemental Fig. 1C) were correlated with the BLI and caliper measurement, which implies that a significant therapeutic response was observed with the combination therapy, followed by chemotherapy and then radiotherapy. The results suggest that a conspicuous antitumor effect of ${ }^{111} \mathrm{In}$-vinorelbine liposomes was achieved via the reduction of the ${ }^{18} \mathrm{~F}-\mathrm{FDG}$ metabolic activity of the tumor. The decreased uptake of ${ }^{18} \mathrm{~F}-\mathrm{FDG}$ may be due to the killing effect of the metabolically viable cells or treatmentinduced necrosis within the tumor.

\section{Body Weight and Survival Assay}

The body weight loss results (Supplemental Fig. 2A) were within the $20 \%$ margin that indicates minimal toxicity of the drug after treatment. The survivals (Supplemental Fig. 2B) were calculated until all mice were dead after injection. The therapeutic response, survival time, and related parameters are summarized in Table 3. The expected growth inhibition rate of the ${ }^{111}$ In-vinorelbine liposomes was $67 \%$. The calculated combination index was $1.06(P<0.01)$. The ${ }^{111}$ In-vinorelbine liposomes increased the median survival time by $35 \mathrm{~d}$ and lengthened the life span by $46 \%$ (log rank, $P=0.0045$ ). No significant difference in the overall survival times between the vinorelbine liposomes and the ${ }^{111} \mathrm{In}$-liposomes was found. The results suggest that the ${ }^{111}$ In-vinorelbine liposomes could display an additive effect for tumor growth suppression, and thus, the results support a possible improvement in overall survival of the treated mice.

\section{Toxicology Studies}

In Table 4, the levels of blood cells of the experimental mice were found to have remained within their reference ranges. The liver and renal function parameters, including ALP, albumin, and BUN of the treated mice, were within or close to their expected reference ranges, even though they were significantly lower than those of the control mice. In addition, the ${ }^{111}$ In-DOTA-treated mice showed a significantly higher serum level of ALT and BUN, suggesting that the liposomal drugs, compared with the unencapsulated form, cause no increase in toxicity.

\section{Histopathologic Examination After Various Treatments}

To investigate the possible toxicities of various treatments, histopathologic examinations of the lungs, liver, spleen, and kidneys, which are the organs found to have higher drug uptake, are shown in Supplemental Figure 3. In addition, the tumor pathologies of various treatments are shown in Supplemental Figure 4. The results show that no significant morphologic damage to most tissues could be detected, except for the kidneys (which had scattered but minimal mononuclear inflammatory cells infiltrated into the interstitium after ${ }^{111}$ In-DOTA treatment). Moreover, the tumor pathology illustrates that the combination therapy had a better therapeutic response in volume control $\left(957 \mathrm{~mm}^{3}\right)$ and displayed a regional necrotic pattern. The larger the tumor size $\left(1,426-2,546 \mathrm{~mm}^{3}\right)$, the greater the level of inherent necrosis observed, which correlates with the BLI results.

\section{DISCUSSION}

PEGylation of the nanoparticle has been reported to play an important role in the stability of nanoparticles. High 


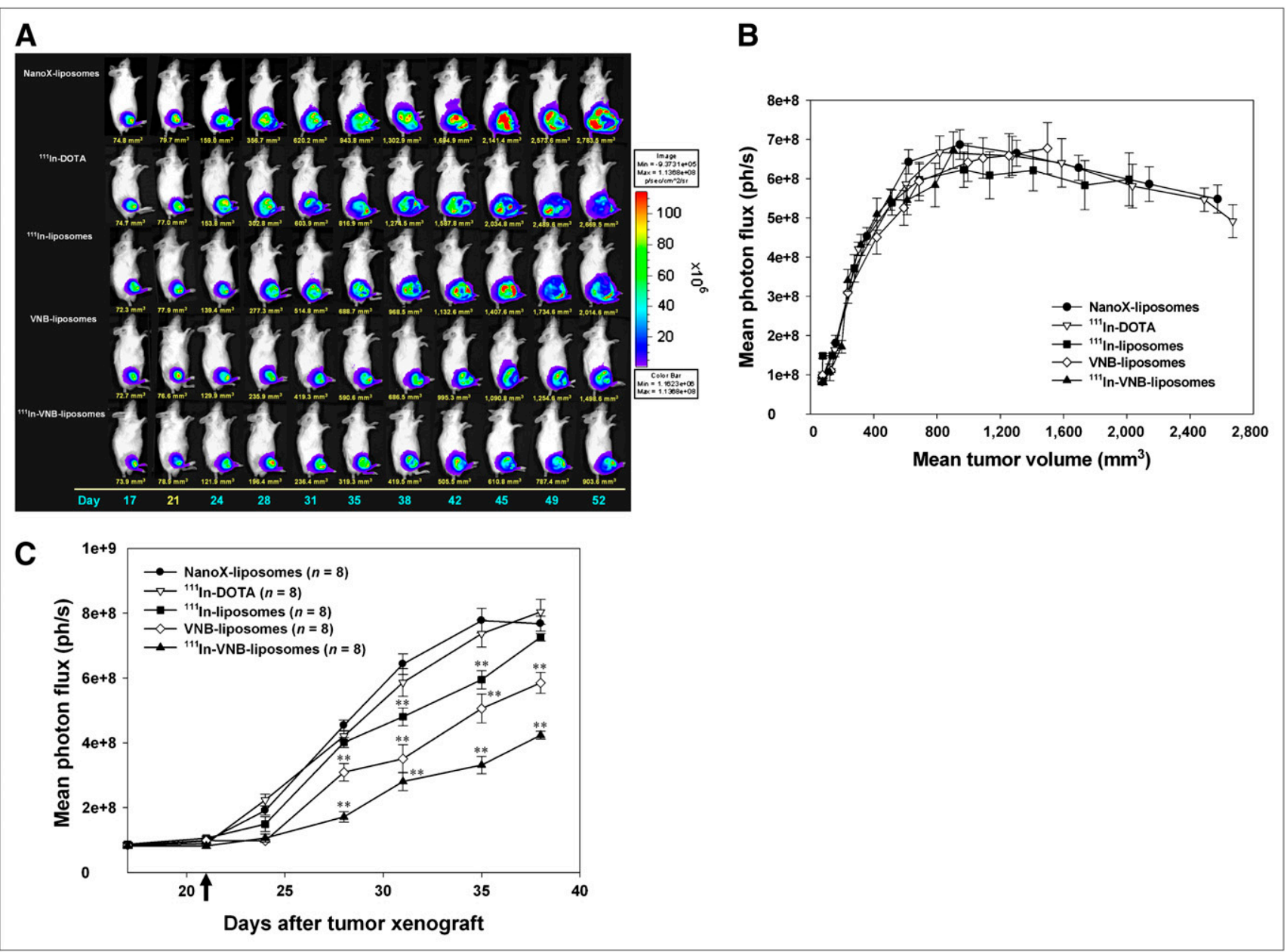

FIGURE 2. (A) In vivo BLI of CT-26/tk-luc tumor-bearing BALB/c mice after various treatments. (B) Mean photon fluxes from ROls of tumor were correlated with tumor size in all treatments. (C) Mean photon flux as function of time after initiation of drug treatment. Combination therapy (i.e., ${ }^{111}$ In-vinorelbine liposomes) had lowest photon collection, which represented best tumor growth suppression. All images were acquired under same experimental conditions and are displayed at same absolute scale. Data are expressed as mean \pm SE. Black arrow indicates time of drug injection. Experiments were repeated twice. ${ }^{\star \star} P<0.01$ is significantly different from control by Student $t$ test. VNB $=$ vinorelbine.

concentration of PEGylation is more able to disturb the balance of both hydrophilicity and hydrophobicity by disrupting the surface integrity of lipid bilayer $(7,20)$. In contrast, a lower concentration of PEGylation increases both the drug uptake level by the RES and the renal elimination rate $(17,21)$. $\mathrm{Li}$ and Huang reported that 5 mol\% PEGylated liposome was optimal as a nanocarrier, judged by the reduction of RES uptake level while the stability of the liposomal structure was maintained (7). Therefore, a suitable level of PEGylation needs to be determined to pinpoint an ideal nanocarrier for the therapeutic efficacy evaluation.

The size of nanoparticle, on the other hand, is directly involved in the drug deposition in tumors. Nanoparticles with a diameter between 100 and $200 \mathrm{~nm}$ have been shown to have a 4-fold higher rate of uptake than do those with

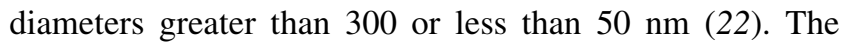
charge status of the nanoliposome also influences its tumor- targeting capability. Negatively charged nanoliposomes ( $\zeta$-potential, $\sim-40 \mathrm{mV}$ ) showed a significantly higher blood clearance rate than that of the neutral nanoliposomes ( $\zeta$-potential, $\sim \pm 10 \mathrm{mV}$ ) because of an acceleration of mononuclear phagocyte system uptake by the liver (23). Positively charged nanoliposomes could cause aggregation with negatively charged serum proteins and result in induced transient embolism of the lung capillaries (24). The PEGylation of nanoliposomes shields the positive or negative charge, turns it to near-neutrality, and results in decreased liver accumulation and prolonged blood circulation (25). To meet these criteria, $6 \mathrm{~mol} \%$ PEGylated liposomes with a diameter of $100 \mathrm{~nm}$ and a $\zeta$-potential of $5.06 \mathrm{mV}$ were used in this study.

The circulation rate was prolonged, and the phagocytic activity of the RES was decreased when 6 mol\% PEG ${ }^{111}$ In-vinorelbine liposomes were used, as shown in Figure 1 and Tables 1 and 2. In addition, the drug uptake in tumor was 


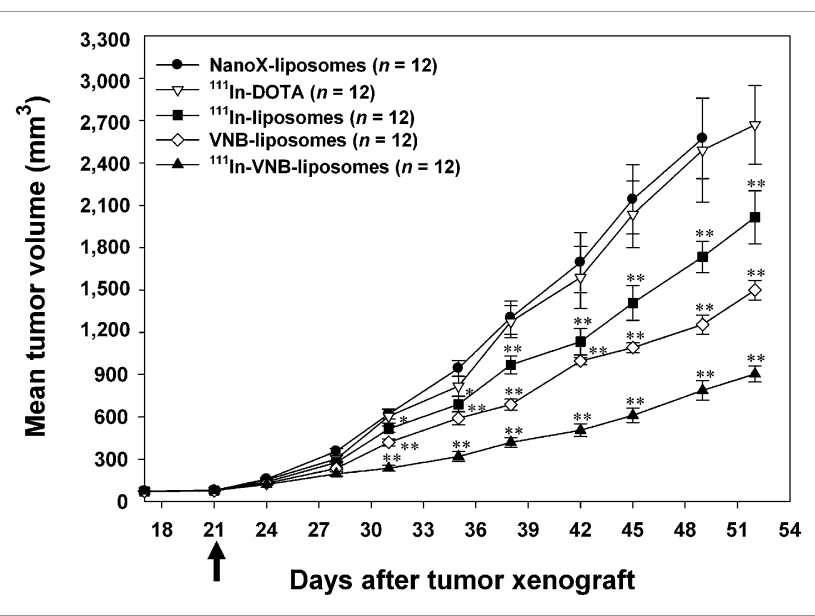

FIGURE 3. Therapeutic responses of CT-26/tk-luc tumorbearing BALB/c mice after various treatments. Greatest tumor growth suppression was found in combination therapy using ${ }^{111} \mathrm{In}$-vinorelbine liposomes, followed by chemotherapy with vinorelbine liposomes, then by radiotherapy with ${ }^{111}$ In-liposomes. Both groups of ${ }^{111}$ In-DOTA and NanoX liposomes are presented as controls for comparison. Data are expressed as mean \pm SE. Experiments were repeated 3 times. ${ }^{\star \star} P<0.01$, Student $t$ test. Black arrow indicates time of drug injection. VNB = vinorelbine.

also improved. The combination effect of ${ }^{111}$ In-vinorelbine liposomes (37 MBq) displayed an additive effect for tumor growth inhibition and overall survival in this preclinical study (Table 3). The toxicologic and histopathologic analyses also revealed that the formulated drug was less toxic to mice (Table 4; Supplemental Fig. 3).

${ }^{18}$ F-FDG PET is well accepted as a gold standard for tumor imaging. Because the oxygen and the nutrient are required for tumor growth, the uptake rate of ${ }^{18} \mathrm{~F}-\mathrm{FDG}$, a radiopharmaceutical of glucose analog, into tissues is proportional to the tumor growth. As such, ${ }^{18} \mathrm{~F}-\mathrm{FDG}$ is a useful indicator for the tumor viability (26). In this study, the uptake of ${ }^{18} \mathrm{~F}$-FDG by the CT-26/tk-luc solid tumor was linearly correlated with tumor size $\left(R^{2}=0.881\right)$ (Supplemental Fig. 1B). ${ }^{18}$ F-FDG uptake as a function of tumor volume showed a tendency to increase (tumor size, $<700$ $\mathrm{mm}^{3}$ ) before reaching a plateau (tumor size, 700-1,200 $\mathrm{mm}^{3}$ ), then increased rapidly (tumor size, $>1,400 \mathrm{~mm}^{3}$ ). The result is similar to that of mean photon flux versus the mean tumor volume obtained from BLI when the tumor size was less than $1,000 \mathrm{~mm}^{3}$ (Fig. 2B); only the SUV curve continued to increase rapidly (tumor size, $>1,400 \mathrm{~mm}^{3}$ ). The difference between the 2 modalities may be due to the characteristics of ${ }^{18} \mathrm{~F}$-FDG PET tumor imaging, which is not capable of thoroughly reflecting the tumor necrosis rate. Alternatively, the tendency of SUV to increase at the later phase of tumor growth may also be due to ${ }^{18} \mathrm{~F}$-FDG uptake by peritumor inflammatory cells (27). Because the diameter of the necrosis center enlarges in parallel with tumor growth, the thickness of the metabolically viable tumor sheath is also increased, as shown in Figure 2A.

Studies using ${ }^{18} \mathrm{~F}-\mathrm{FDG}$ PET suggest that a decrease in SUV could be a predictive assay for therapeutic response in malignant lymphoma and a variety of solid tumors (28). Metabolic activities using ${ }^{18} \mathrm{~F}-\mathrm{FDG}$ PET for all treatments in this study were also shown to increase continuously as tumors grew (Supplemental Fig. 1C). The combination therapy with ${ }^{111}$ In-vinorelbine liposomes displayed a detectable effect for tumor growth inhibition via the reduction of ${ }^{18} \mathrm{~F}-\mathrm{FDG}$ metabolism. This result is similar to the one reported in the study by Oya et al.: that a marked decrease in ${ }^{18} \mathrm{~F}-\mathrm{FDG}$ uptake corresponded to a reduced number of tumor cells (29). For tumor pathologic response, no quantitative correlation between decreased SUV and the tumor necrosis rate was found at the end of the treatments. In summary, the application of ${ }^{18} \mathrm{~F}$-FDG PET in monitoring tumor therapeutic response in preclinical studies should take into account the possible effects of treatment-induced inflammation and tumor necrosis.

\section{TABLE 3. Tumor Growth Inhibition and Survival Time of CT-26/tk-luc Tumor-Bearing BALB/c Mice $(n=12)$ After}

Intravenous Administration of Different Treatments

\begin{tabular}{|c|c|c|c|c|c|c|c|}
\hline \multirow[b]{2}{*}{ Treatment } & \multicolumn{4}{|c|}{ Tumor growth inhibition } & \multicolumn{3}{|c|}{ Survival time } \\
\hline & $\begin{array}{l}\text { Mean growth } \\
\text { inhibition } \\
\text { rate }(\%)\end{array}$ & $\begin{array}{l}\text { Expected growth } \\
\text { inhibition } \\
\text { rate }(\%)\end{array}$ & $\begin{array}{l}\text { Combination } \\
\text { index }\end{array}$ & $P$ & $\begin{array}{l}\text { Median } \\
\text { survival } \\
\text { time (d) }\end{array}$ & $\begin{array}{l}\text { Increase } \\
\text { in life } \\
\text { span }(\%)^{\star}\end{array}$ & $P$ \\
\hline${ }^{111}$ In-vinorelbine liposomes & 69 & 67 & 1.06 & $<0.01$ & 35 & +46 & 0.0045 \\
\hline Vinorelbine liposomes & 51 & - & - & $<0.01$ & 31 & +29 & 0.0136 \\
\hline${ }^{111}$ In-liposomes & 33 & - & - & $<0.01$ & 31 & +29 & 0.0083 \\
\hline${ }^{111}$ In-DOTA & 3 & - & - & NS & 28 & +17 & NS \\
\hline NanoX liposomes & - & - & - & - & 24 & - & - \\
\hline
\end{tabular}


TABLE 4. Biochemical and Hematologic Analyses of CT-26/tk-luc Tumor-Bearing BALB/c Mice

at Fifth Week After Different Treatments

\begin{tabular}{|c|c|c|c|c|c|c|}
\hline \multirow[b]{2}{*}{ Parameter } & \multicolumn{6}{|c|}{ Mean value after administration of $35 \mathrm{~d}$} \\
\hline & $\begin{array}{l}{ }^{111} \text { In-vinorelbine } \\
\text { liposome }\end{array}$ & ${ }^{111}$ In-liposome & ${ }^{111}$ In-DOTA & $\begin{array}{l}\text { Vinorelbine } \\
\text { liposome }\end{array}$ & $\begin{array}{c}\text { NanoX } \\
\text { liposome }\end{array}$ & $\begin{array}{l}\text { Reference } \\
\text { range }\end{array}$ \\
\hline \multicolumn{7}{|l|}{ Blood cells } \\
\hline $\begin{array}{l}\text { WBCs } \\
\qquad\left(10^{3} / \mu L\right)\end{array}$ & $6.83 \pm 0.27$ & $6.12 \pm 0.21$ & $7.32 \pm 0.34$ & $8.99 \pm 1.01$ & $5.62 \pm 0.12$ & $3.2-12.7$ \\
\hline $\begin{array}{l}\text { RBCs } \\
\left(10^{6} / \mu \mathrm{L}\right)\end{array}$ & $7.78 \pm 0.35$ & $8.23 \pm 0.32$ & $7.86 \pm 0.73$ & $8.30 \pm 0.91$ & $10.23 \pm 0.85$ & $7.0-10.1$ \\
\hline HGB (g/L) & $12.30 \pm 1.25$ & $11.43 \pm 1.31$ & $12.96 \pm 1.98$ & $12.40 \pm 1.54$ & $12.53 \pm 1.56$ & $11.8-14.9$ \\
\hline $\mathrm{HCT}$ & $37.50 \pm 1.54$ & $37.34 \pm 1.97$ & $38.54 \pm 2.23$ & $37.27 \pm 2.51$ & $40.31 \pm 3.58$ & $36.7-46.8$ \\
\hline $\begin{array}{l}\text { PLTs } \\
\qquad\left(10^{3} / \mu L\right)\end{array}$ & $1,312.21 \pm 121.12$ & $1,583.45 \pm 211.45$ & $1,487.56 \pm 119.03$ & $1,433.5 \pm 107.88$ & $1,116.23 \pm 98.76$ & $766-1,657$ \\
\hline \multicolumn{7}{|l|}{ Serum } \\
\hline \multicolumn{7}{|l|}{ Liver function } \\
\hline $\operatorname{ALP}(\mathrm{U} / \mathrm{L})$ & $70.5 \pm 4.21^{*}$ & $72.27 \pm 5.67^{*}$ & $150.71 \pm 7.96$ & $89.12 \pm 10.23^{*}$ & $112.24 \pm 12.56$ & $62-209$ \\
\hline Albumin $(\mathrm{g} / \mathrm{dL})$ & $2.40 \pm 0.02^{*}$ & $2.40 \pm 0.01^{\star}$ & $2.81 \pm 0.06$ & $2.4 \pm 0.01^{*}$ & $2.73 \pm 0.01$ & $2.5-4.8$ \\
\hline $\operatorname{ALT}(\mathrm{U} / \mathrm{L})$ & $29.21 \pm 1.23$ & $29.89 \pm 1.20$ & $77.86 \pm 7.33^{\dagger}$ & $37.69 \pm 3.54$ & $28.94 \pm 2.54$ & $28-132$ \\
\hline AST (U/L) & $219.00 \pm 42.45$ & $224.12 \pm 45.89$ & $131.86 \pm 12.41$ & $227.32 \pm 24.21$ & $145.67 \pm 19.84$ & $59-247$ \\
\hline \multicolumn{7}{|l|}{ Kidney function } \\
\hline Creatinine $(\mathrm{mg} / \mathrm{dL})$ & $0.25 \pm 0.05$ & $0.23 \pm 0.07$ & $0.29 \pm 0.01$ & $0.23 \pm 0.06$ & $0.24 \pm 0.07$ & $0.2-0.8$ \\
\hline BUN (mg/dL) & $17.05 \pm 0.12^{*}$ & $24.1 \pm 0.32$ & $35.29 \pm 1.66^{*}$ & $19.90 \pm 1.21^{*}$ & $27.57 \pm 2.32$ & $18-29$ \\
\hline \multicolumn{7}{|c|}{$\begin{array}{l}\text { WBCs }=\text { white blood cells; RBCs }=\text { red blood cells; HGB }=\text { hemoglobin; HCT = hematocrit; PLTs }=\text { platelets; } A S T=\text { aspartate } \\
\text { aminotransferase. } \\
\text { Data are expressed as mean } \pm \text { SE from } 5 \text { mice administered } 37 \mathrm{MBq} \text { of }{ }^{111} \mathrm{In} \text {-vinorelbine liposomes, }{ }^{111} \mathrm{In} \text {-liposomes, }{ }^{111} \mathrm{In} \text {-DOTA, or } \\
\text { equivalent amount of unlabeled vinorelbine liposomes. }\end{array}$} \\
\hline
\end{tabular}

\section{CONCLUSION}

Liposomes modified with 6 mol\% PEGylation were shown to have higher tumor uptake but lower accumulation in the RES than those modified with 0.9 mol\% PEGylation. The passive nanotargeted $6 \mathrm{~mol} \%$ PEG ${ }^{111}$ In-vinorelbine liposomes displayed an additive effect for tumor growth suppression, not only reducing tissue toxicity but also improving survivability during these therapeutic trials. The results suggest that this formulation of the nanomedicine may have potential, when combined with noninvasive molecular imaging, for the study of tumor targeting, localization, and bioavailability in preclinical studies.

\section{ACKNOWLEDGMENTS}

We thank the Molecular-Genetic Imaging Core/NRPGM, Taiwan, for its support. This study was supported by grant 97A1-NMPPO1-007 from the National Health Research Institute, Taipei, Taiwan. The nanoliposomes were kindly provided by Taiwan Liposome Company, Taipei, Taiwan.

\section{REFERENCES}

1. Torchilin VP. Recent advances with liposomes as pharmaceutical carriers. Nat Rev Drug Discov. 2005;4:145-160.

2. Allen TM, Cullis PR. Drug delivery systems: entering the mainstream. Science. 2004;303:1818-1822.
3. Chow TH, Lin YY, Hwang JJ, et al. Diagnostic and therapeutic evaluation of ${ }^{111}$ In-vinorelbine-liposomes in a human colorectal carcinoma HT-29/luc-bearing animal model. Nucl Med Biol. 2008;35:623-634.

4. Bakker-Woudenberg IA, Lokerse AF, Roerdink FH. Antibacterial activity of liposome-entrapped ampicillin in vitro and in vivo in relation to the lipid composition. J Pharmacol Exp Ther. 1989;251:321-327.

5. Messerer CL, Ramsay EC, Waterhouse D, et al. Liposomal irinotecan: formulation development and therapeutic assessment in murine xenograft models of colorectal cancer. Clin Cancer Res. 2004;10:6638-6649.

6. Boerman OC, Storm G, Oyen WJ, et al. Sterically stabilized liposomes labeled with indium-111 to image focal infection. J Nucl Med. 1995;36:1639-1644.

7. Li SD, Huang L. Pharmacokinetics and biodistribution of nanoparticles. Mol Pharm. 2008:5:496-504

8. Udhrain A, Skubitz KM, Northfelt DW. Pegylated liposomal doxorubicin in the treatment of AIDS-related Kaposi's sarcoma. Int J Nanomedicine. 2007;2:345-352.

9. Northfelt DW, Dezube BJ, Thommes JA, et al. Pegylated-liposomal doxorubicin versus doxorubicin, bleomycin, and vincristine in the treatment of AIDS-related Kaposi's sarcoma: results of a randomized phase III clinical trial. J Clin Oncol. 1998; 16:2445-2451.

10. Green AE, Rose PG. Pegylated liposomal doxorubicin in ovarian cancer. Int $J$ Nanomedicine. 2006;1:229-239.

11. Gordon AN, Fleagle JT, Guthrie D, Parkin DE, Gore ME, Lacave AJ. Recurrent epithelial ovarian carcinoma: a randomized phase III study of pegylated liposomal doxorubicin versus topotecan. J Clin Oncol. 2001;19:3312-3322.

12. Minisini AM, Andreetta C, Fasola G, Puglisi F. Pegylated liposomal doxorubicin in elderly patients with metastatic breast cancer. Expert Rev Anticancer Ther. 2008;8:331-342.

13. Harris L, Batist G, Belt R, et al. Liposome-encapsulated doxorubicin compared with conventional doxorubicin in a randomized multicenter trial as first-line therapy of metastatic breast carcinoma. Cancer. 2002;94:25-36.

14. Katsumata N, Fujiwara Y, Kamura T, et al. Phase II clinical trial of pegylated liposomal doxorubicin (JNSO02) in Japanese patients with mullerian carcinoma (epithelial ovarian carcinoma, primary carcinoma of fallopian tube, peritoneal carcinoma) having a therapeutic history of platinum-based chemotherapy: 
a Phase II Study of the Japanese Gynecologic Oncology Group. Jpn J Clin Oncol. 2008;38:777-785.

15. Gabizon A, Shmeeda H, Barenholz Y. Pharmacokinetics of pegylated liposomal Doxorubicin: review of animal and human studies. Clin Pharmacokinet. 2003; 42:419-436.

16. Heidenreich A, Sommer F, Ohlmann CH, et al. Prospective randomized phase II trial of pegylated doxorubicin in the management of symptomatic hormonerefractory prostate carcinoma. Cancer. 2004;101:948-956.

17. Chow TH, Lin YY, Hwang JJ, et al. Improvement of biodistribution and therapeutic index via increase of polyethylene glycol on drug-carrying liposomes in an HT-29/luc xenografted mouse model. Anticancer Res. 2009;29:2111-2120.

18. Tseng YL, Hong RL, Tao MH, Chang FH. Sterically stabilized anti-idiotype immunoliposomes improve the therapeutic efficacy of doxorubicin in a murine B-cell lymphoma model. Int J Cancer. 1999;80:723-730.

19. Chen LC, Chang $\mathrm{CH}$, Yu CY, et al. Biodistribution, pharmacokinetics and imaging of ${ }^{188}$ Re-BMEDA-labeled pegylated liposomes after intraperitoneal injection in a C26 colon carcinoma ascites mouse model. Nucl Med Biol. 2007;34:415-423.

20. Dos Santos N, Allen C, Doppen AM, et al. Influence of poly(ethylene glycol) grafting density and polymer length on liposomes: relating plasma circulation lifetimes to protein binding. Biochim Biophys Acta. 2007;1768:13671377.
21. Lee WC, Hwang JJ, Tseng YL, et al. Therapeutic efficacy evaluation of ${ }^{111} \mathrm{In}$ VNB-liposome on human colorectal adenocarcinoma HT-29/luc mouse xenografts. Nucl Instrum Meth Phys Res A. 2006;569:497-504.

22. Liu D, Mori A, Huang L. Role of liposome size and RES blockade in controlling biodistribution and tumor uptake of GM1-containing liposomes. Biochim Biophys Acta. 1992;1104:95-101.

23. Torchilin VP, Lukyanov AN, Gao Z, Papahadjopoulos-Sternberg B. Immunomicelles: targeted pharmaceutical carriers for poorly soluble drugs. Proc Natl Acad Sci USA. 2003;100:6039-6044.

24. Zhang JS, Liu F, Huang L. Implications of pharmacokinetic behavior of lipoplex for its inflammatory toxicity. Adv Drug Deliv Rev. 2005;57:689-698.

25. Levchenko TS, Rammohan R, Lukyanov AN, Whiteman KR, Torchilin VP. Liposome clearance in mice: the effect of a separate and combined presence of surface charge and polymer coating. Int J Pharm. 2002;240:95-102.

26. Oya N, Nagata Y, Ishigaki T, et al. Evaluation of experimental liver tumors using fluorine-18-2-fluoro-2-deoxy-D-glucose PET. J Nucl Med. 1993;34:2124-2129.

27. Spaepen K, Stroobants S, Dupont P, et al. $\left[{ }^{18} \mathrm{~F}\right] \mathrm{FDG}$ PET monitoring of tumour response to chemotherapy: does $\left[{ }^{18} \mathrm{~F}\right] \mathrm{FDG}$ uptake correlate with the viable tumour cell fraction? Eur J Nucl Med Mol Imaging. 2003;30:682-688.

28. Juweid ME, Cheson BD. Positron-emission tomography and assessment of cancer therapy. N Engl J Med. 2006;354:496-507.

29. Oya N, Nagata Y, Tamaki N, et al. FDG-PET evaluation of therapeutic effects on VX2 liver tumor. J Nucl Med. 1996;37:296-302. 